\title{
Toxicity assessment of treated sewage effluent using the Zebrafish embryo model
}

\author{
Zain Z. Zakaria ${ }^{1}$, Halah N. Nasir, Fatima Nasser, Huseyin C Yalcin ${ }^{1}$ \\ ${ }^{1}$ Biomedical Research Center, Qatar University, Doha, QATAR \\ ${ }^{2}$ College of Health Science, Qatar University, Doha, QATAR
}

\section{BACKGROUND}

In a context of tremendous economic value, the management and protection of water resources in Qatar has long been a significant issue as part of the global wastewater management plan. The process is based on several stages of treatment in order to deliver high-quality effluent standard. Treated sewage effluent (TSE) can potentially be used for agriculture in Qatar and it should be biologically evaluated before releasing it to the environment. TSE water can be further filtered with techniques that include forward osmosis FO, reverse osmosis RO, and nanofiltration NF.

\section{OBJECTIVE}

This main objective of this project to assess the toxicity of differently treated sewage effluent water on the environment using the zebrafish model. Our approach will also be relevant to the assessment of the water quality for agriculture use.

\section{METHODOLOGY}

\section{I.Toxicity Assessment}

Survival Rate: Embryos incubated at $28 \mathrm{dc}$ and number of deaths recorded at $24 \mathrm{hpf}, 48 \mathrm{hpf}$ and $72 \mathrm{hpf}$.

Tail Flicking: Locomotive activity of the embryos. Analysis done using DanioScope software.

Hatching Rate: \% of hatched embryos out of the total viable embryos calculated at $48 \mathrm{hpf}$.

Locomotion assays: to reveal if there potential neuro/muscular effect at $72 \mathrm{hpf}$ developing embryos.

\section{Cardio toxicity Assessment}

Blood Flow Analysis: Analysis of blood flow and tracking red blood cell movement

Heart Structure Analysis: Size of the cardiac chambers and the myocardial wall.

\section{Molecular Techniques}

RNA Extraction: to detect gene expression.

cDNA synthesis

Quantitative real time polymerase chain reaction (RT-qPCR)

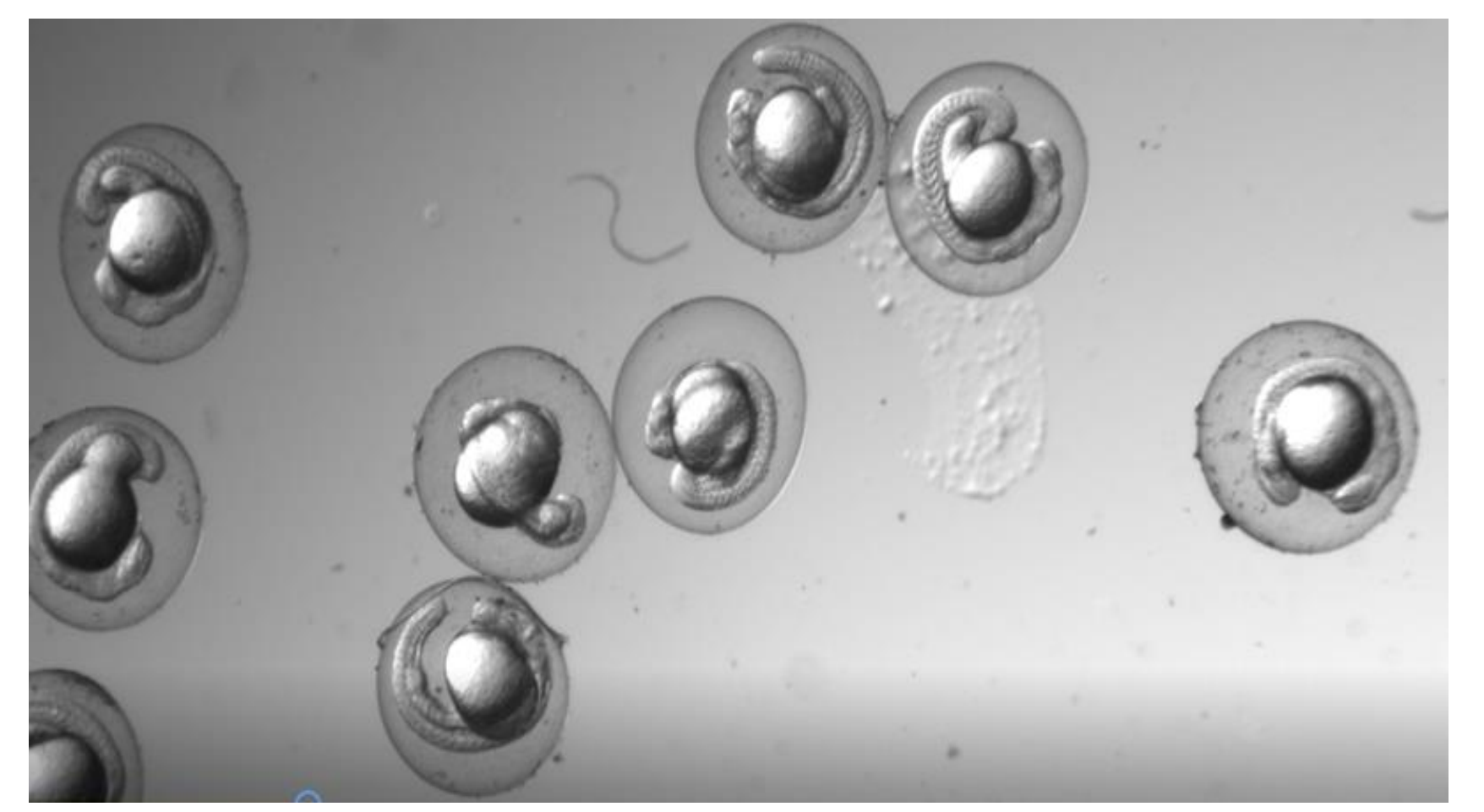

Figure (1): Tail flicking activity at $24 \mathrm{hpf}$

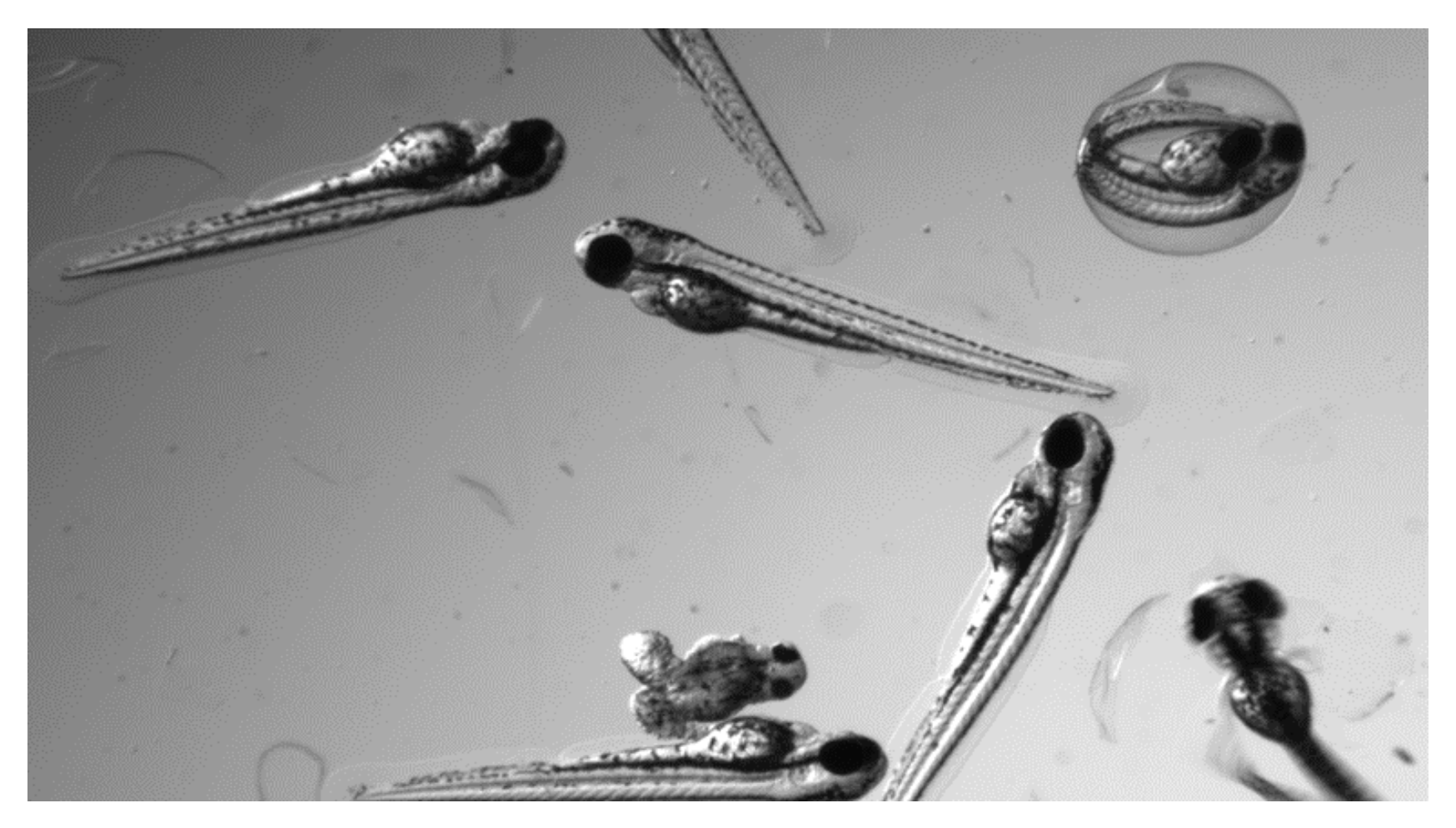

Figure (2): Hatching rate at $48 \mathrm{hpf}$

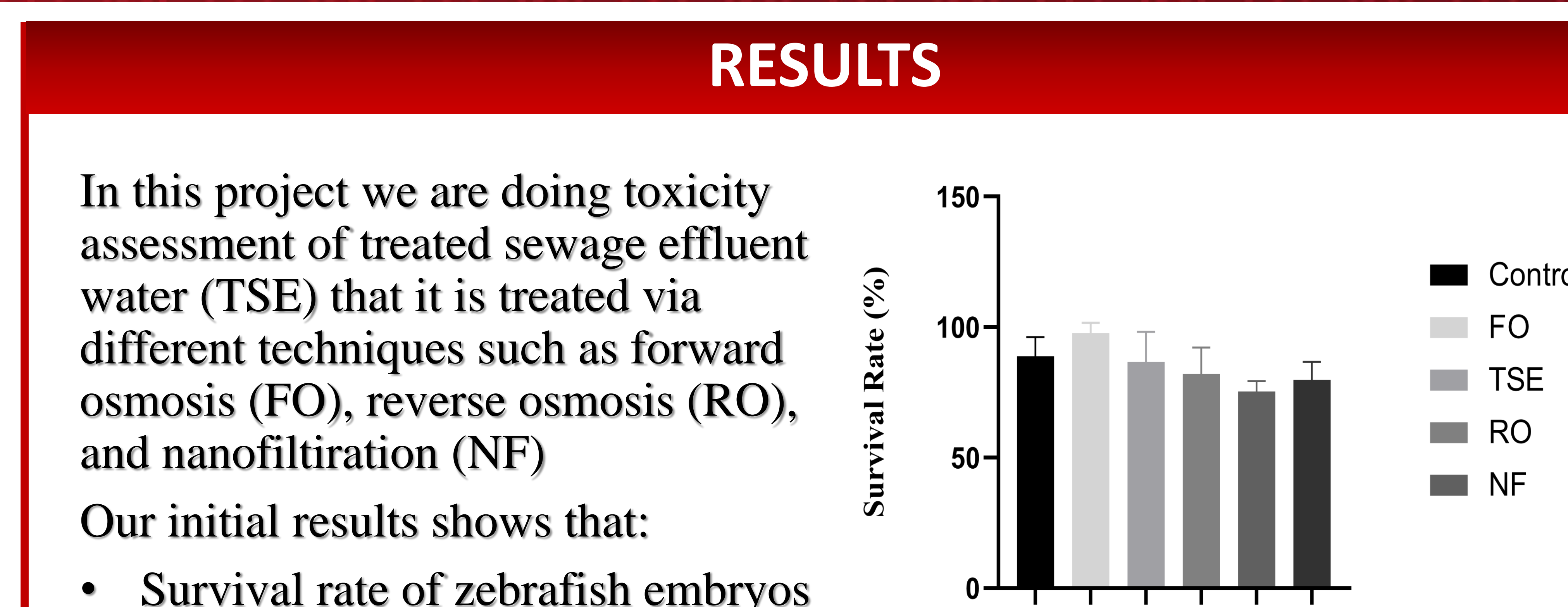

Survival rate of zebrafish embryos was not affected significantly when Figure (3): Survival rate of control (egg water), cultured in different water samples System water ( for adult fish husbandry), forward - (figure 3). osmosis (FO), treated sewage effluent (TSE), Reverse osmosis (RO), and nanofiltration (NF) at 24hpf.
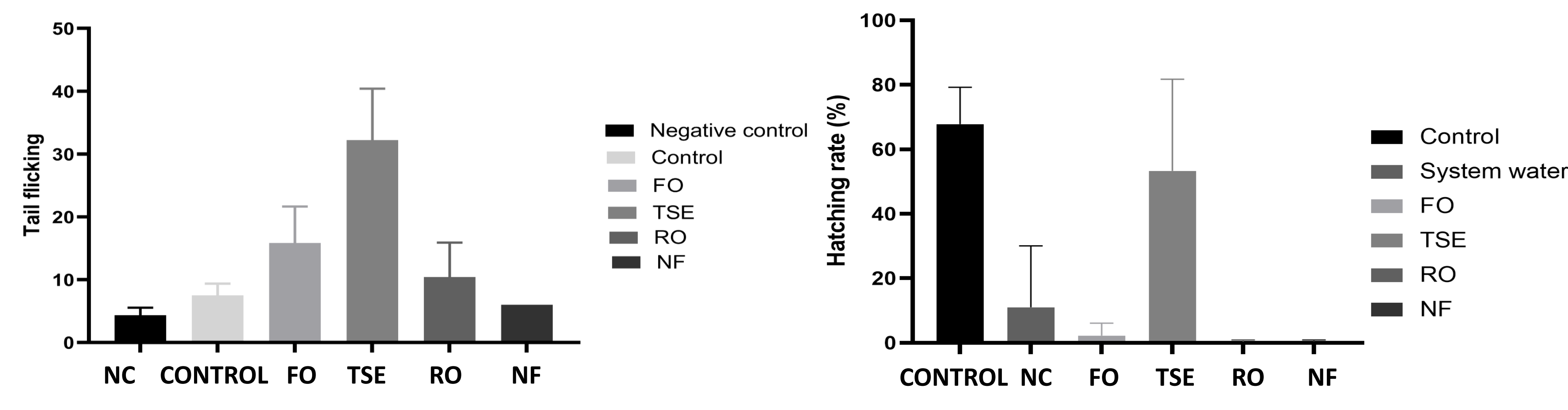

Figure (4): Tail flicking activity at $24 \mathrm{hpf}$
- Significant increase in burst count/ minute(Tail flicking activity)caused by Forward osmosis and treated sewage effluent samples due to high total dissolved substance (TDS) in these samples (figure 4).

- Significantly reduced hatching rate ability for treated embryos with System water, FO, RO, NF water samples (figure 5).
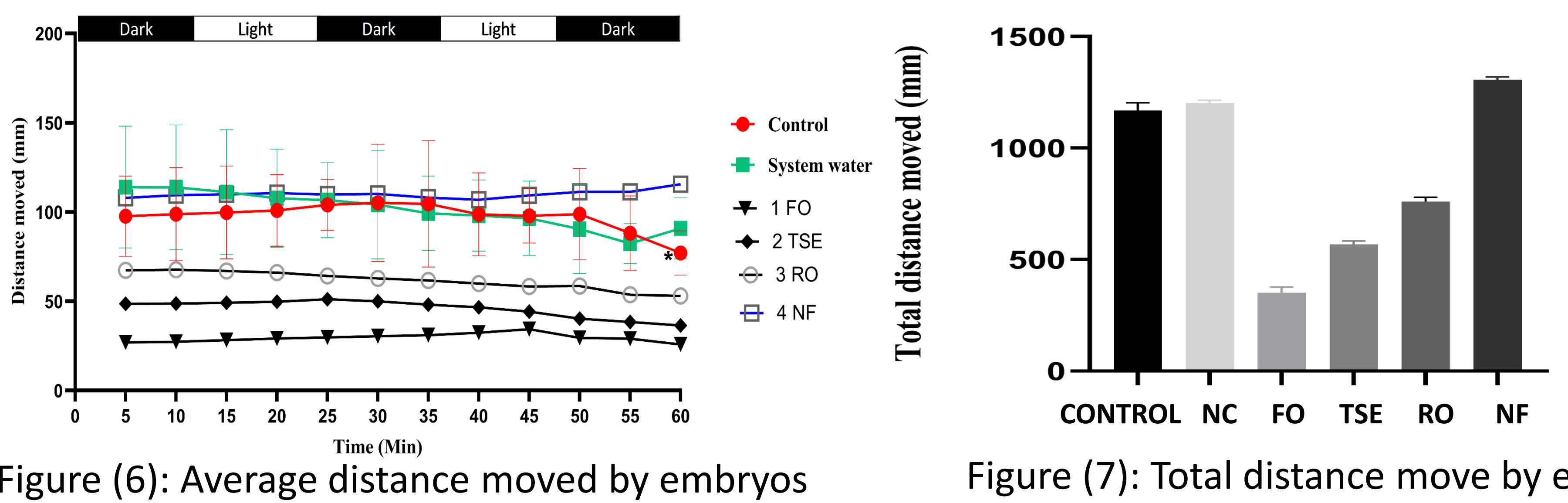

Figure (7): Total distance move by embryos

- The locomotion assays revealed potential neuro/muscular effect at $72 \mathrm{hpf}$ developing embryos treated with FO, TES, RO water sample (figure 6).
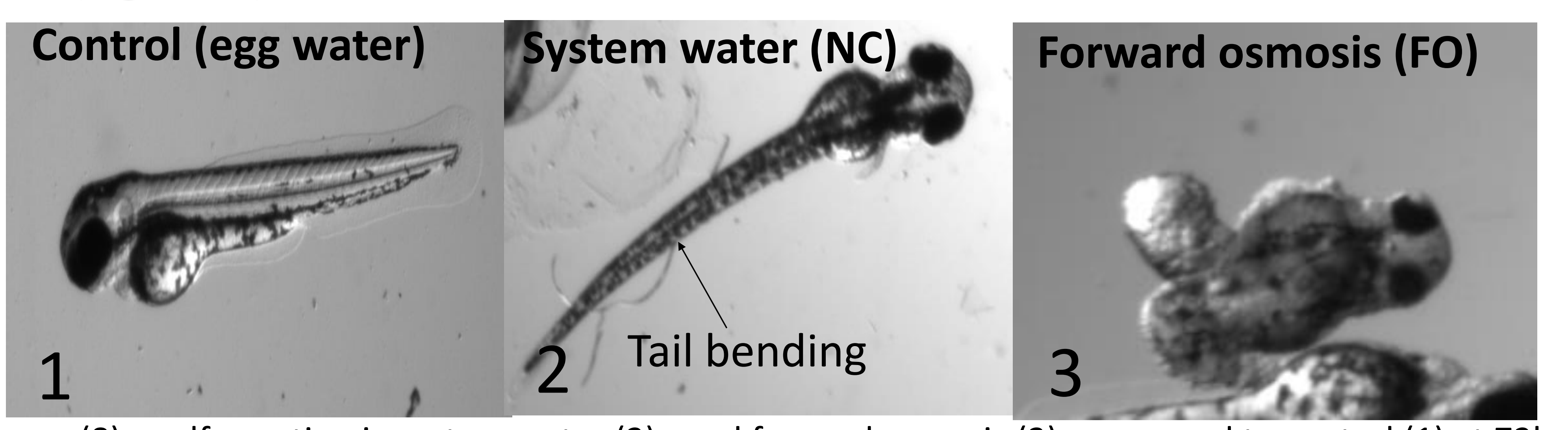

Figure (8): malformation in system water (2), and forward osmosis (3), compared to control (1) at $72 \mathrm{hp}$

- Developmental defects and Malformation in shape in different water sample appear (system water) figure (2), and forward osmosis figure (3) at 72hpf. compared to control (figure 8).

\section{CONCLUSION}

Utilization of TSE for environmental and agricultural purposes will have an economical value in the nation. It is critically important to determine the most efficient and less toxic ways of water filtration. Zebrafish is a practical model that can be used to assess water toxicity. This project aims to examine toxicity of effluent water filtration techniques using the zebrafish model. Our results suggest that there is a developmental effect of the treatment to the animal that we can see in toxicity and morphological assessment. Further testing is ongoing to confirm the results 